\title{
NUEVO CENTRO COMERCIAL EN CAMPANAR/VALENCIA/ESPAÑA
}

\author{
(NEW SHOPPING CENTRE IN CAMPANAR/VALENCIA/SPAIN) \\ Fernando M. García-Ordóñez, Dr. Arquitecto. Director del Proyecto
}

\section{RESUMEN}

Se describen en este articulo las características de un Centro Comercial ubicado en el Polígono de Campanar de Valencia. Este Centro se compone de cuatro niveles perfectamente definidos:

- Planta - 4 (comercial).

- Planta 0 (comercial).

- Planta + 4 (aparcamiento).

- Planta +7 (aparcamiento).

Dentro de estos niveles, las áreas comerciales se distribuyen entre dos grandes almacenes y tiendas.

\section{SUMMARY}

This work describes the characteristics of a Shopping Centre located in Campanar Area, in Valencia. This Centre include four perfectly defined levels:

- Floor -4 (shopping)

- Floor 0 (shopping).

- Floor +4 (parking).

- Floor +7 (parking).

Within these levels two general stores and shops share the commercial area.

\section{INTRODUCCION}

En este Complejo Comercial constan los siguientes edificios (ver esquema):

\section{Area comercial (1)}

Está constituida por dos plantas comerciales servidas por una gran «calle» con huecos destinados a interpenetración entre ambas plantas, mediante escaleras mecánicas. Sobre estas

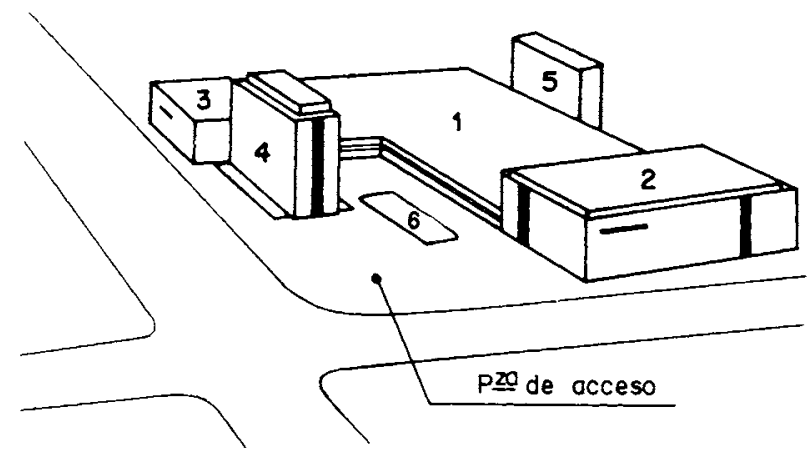

1.-Area comercial y aparcamiento en cubierta. 2.-Gran almacén (5 plantas). 3.-Gran almacén (5 plantas). 4.-Hotel. 5.-Oficinas. 6.-Patio de la planta de las escaleras de bajada. plantas existen otras dos que sirven de aparcamiento unidas, a su vez, al área comercial, también mediante escaleras mecánicas. La "calle» comercial, en forma de L, tiene en sus dos extremos sendos grandes almacenes de cinco plantas cada uno. La plaza de acceso, envuelta por la $L$ comercial, dispone de un hueco para penetración visual y peatonal (una escalera y una rampa) hacia la planta comercial -4 , que se extiende también bajo la citada plaza de acceso. Los aparcamientos, con capacidad para 2.000 plazas, tienen acceso por medio de una rampa helicoidal y la salida a través de una rampa recta. Disponen asimismo de escaleras mecánicas.

\section{Grandes almacenes (2 y 3)}

Como se desprende del texto anterior, los dos grandes almacenes quedan incorporados al espacio comercial general (1) a través de la «calle» interior.

\section{Hotel y oficinas (4 y 5 )}

Ambos edificios disponen de accesos independientes, aunque se encuentran unidos al Conjunto Comercial por medio de la gran plaza de acceso y la calle comercial. 


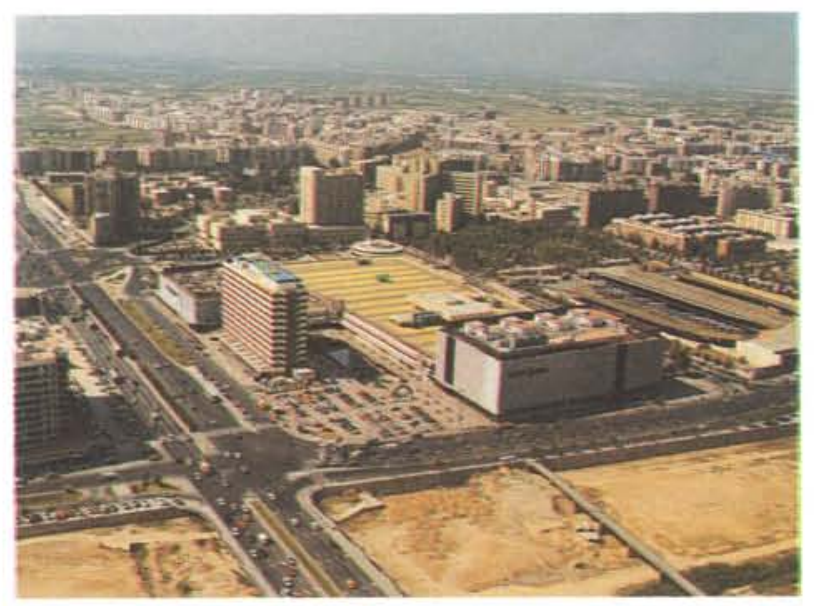

El solar donde se ubica el Nuevo Centro de Valencia posee una superficie total de $50.058 \mathrm{~m}^{2}$ alcanzándose, al final de las obras y según la Normativa Urbanistica existente, una superficie construida total de $112.780 \mathrm{~m}^{2}$ desglosada del modo siguiente:

\author{
$82.500 \mathrm{~m}^{2}$ Uso Comercial (grandes almacentes, Galeria co- \\ mercial, cafeterias, espectáculos, etc.). \\ $13.300 \mathrm{~m}^{2}$ Hotel y comercios. \\ $13.000 \mathrm{~m}^{2}$ Oficinas, negocios y comercios. \\ $4.000 \mathrm{~m}^{2}$ Exposiciones, muestras y servicios. \\ $112.800 \mathrm{~m}^{2}$ TOTAL.
}

Sin incluir aparcamientos $\left(75.000 \mathrm{~m}^{2}\right)$ y otros espacios no $\mathrm{cu}$ biertos; corredores, accesos, etc. $\left(18.600 \mathrm{~m}^{2}\right)$.

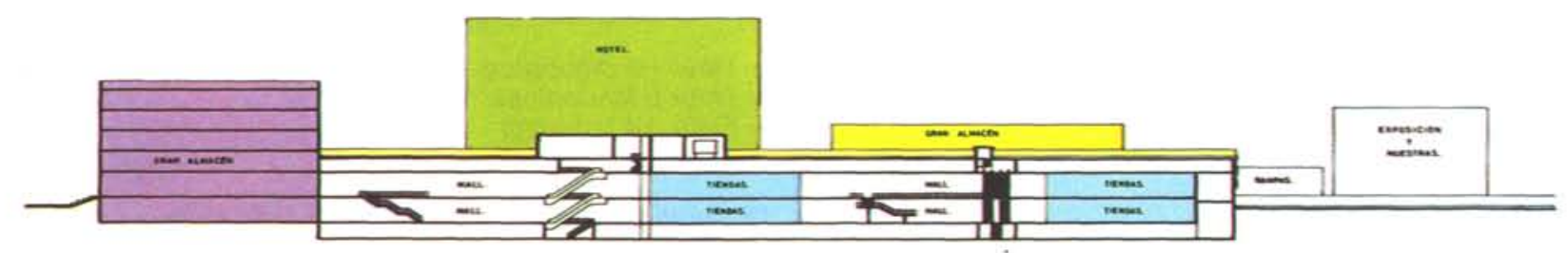

Sección longitudinal.

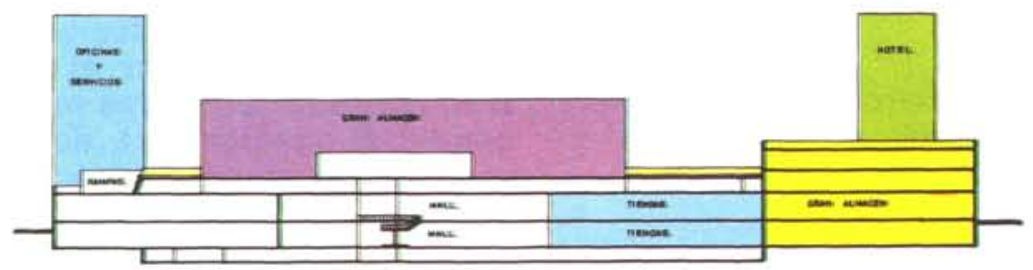

Sección transversal.

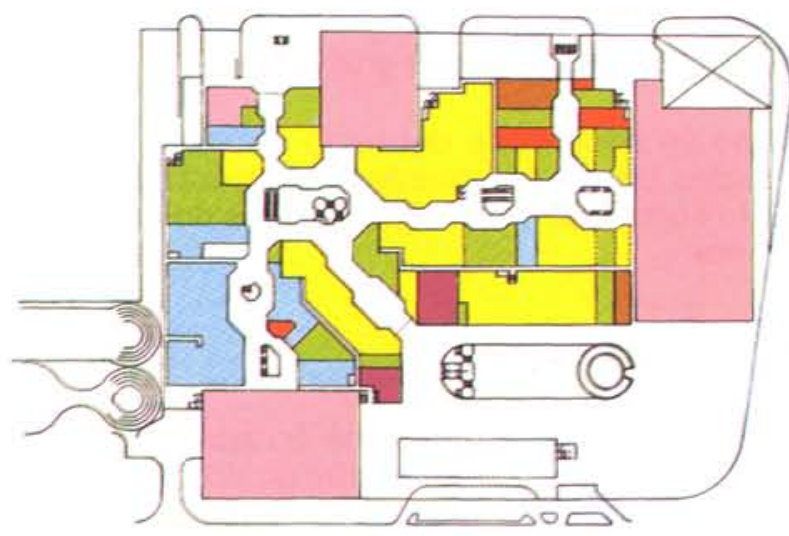

Nivel superior. (c) Consejo Superior de Investigaciones Científicas Licencia Creative Commons 3.0 España (by-nc)

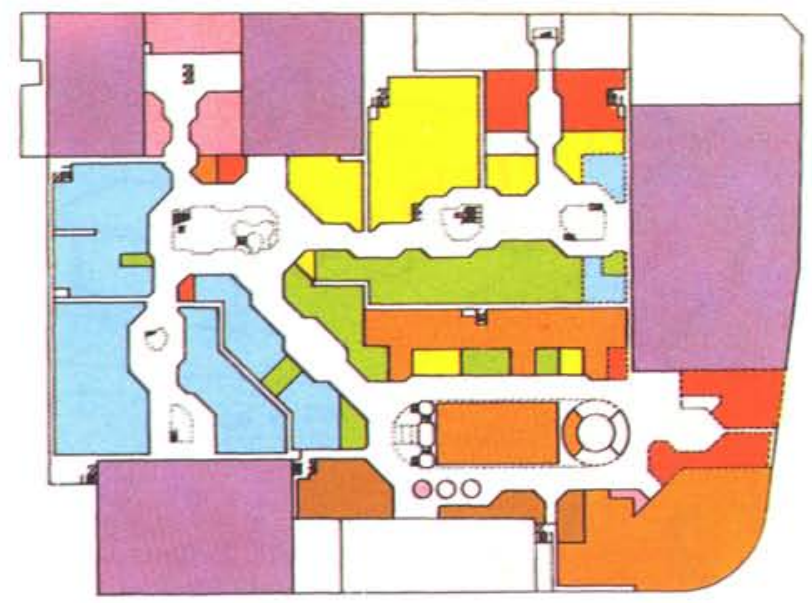

Nivel inferior.

http://informesdelaconstruccion.revistas.csic.es 


\section{ESTRUCTURAS}

\section{Justificación de la solución adoptada}

Dada la importancia del Centro y la gran repercusión que el coste de la estructura tiene en el conjunto, se ha realizado un estudio de distintas soluciones que detallamos:

a) Estructura octogonal plana aligerada.

b) Estructura en losa plana de canto uniforme.

c) Estructura con vigas de gran canto y forjados varios.

d) Estructura con vigas planas y forjado de losa.

e) Estructura con vigas planas de sección variable y losa.

La solución "C" fue desechada por el problema que las vigas ocasionaban en la distribución de instalaciones.

La solución "a» que es la que aparentemente utiliza menos materiales, se rechazó por el incre-

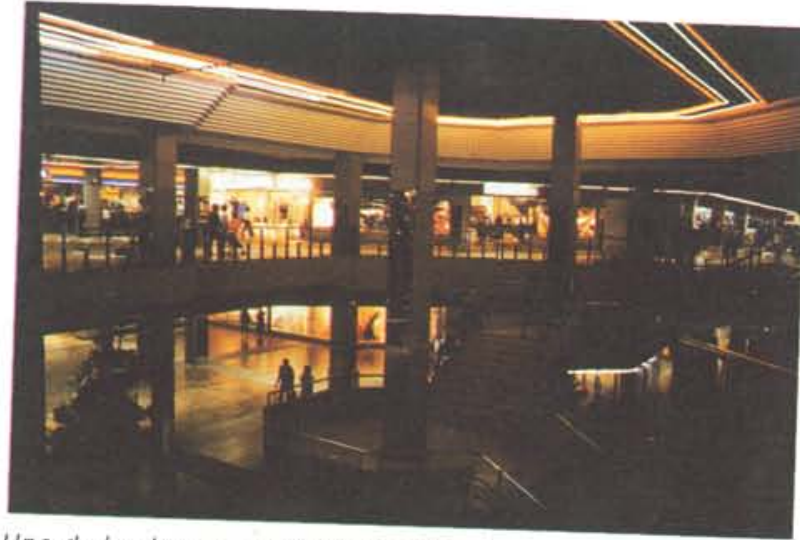

Uno de los huecos de interpenetración.

mento de costo que supone la utilización de los casetones y la complicación en la ejecución de la ferralla.

De entre las soluciones «b», «d" $y$ «e», una vez realizado un cálculo detallado de módulos tipo y aplicando a cada uno de los elementos el costo actual de mercado, se consideró como más conveniente, desde el punto de vista económicofuncional, la de vigas planas con sección cons. tante y forjado de losa.

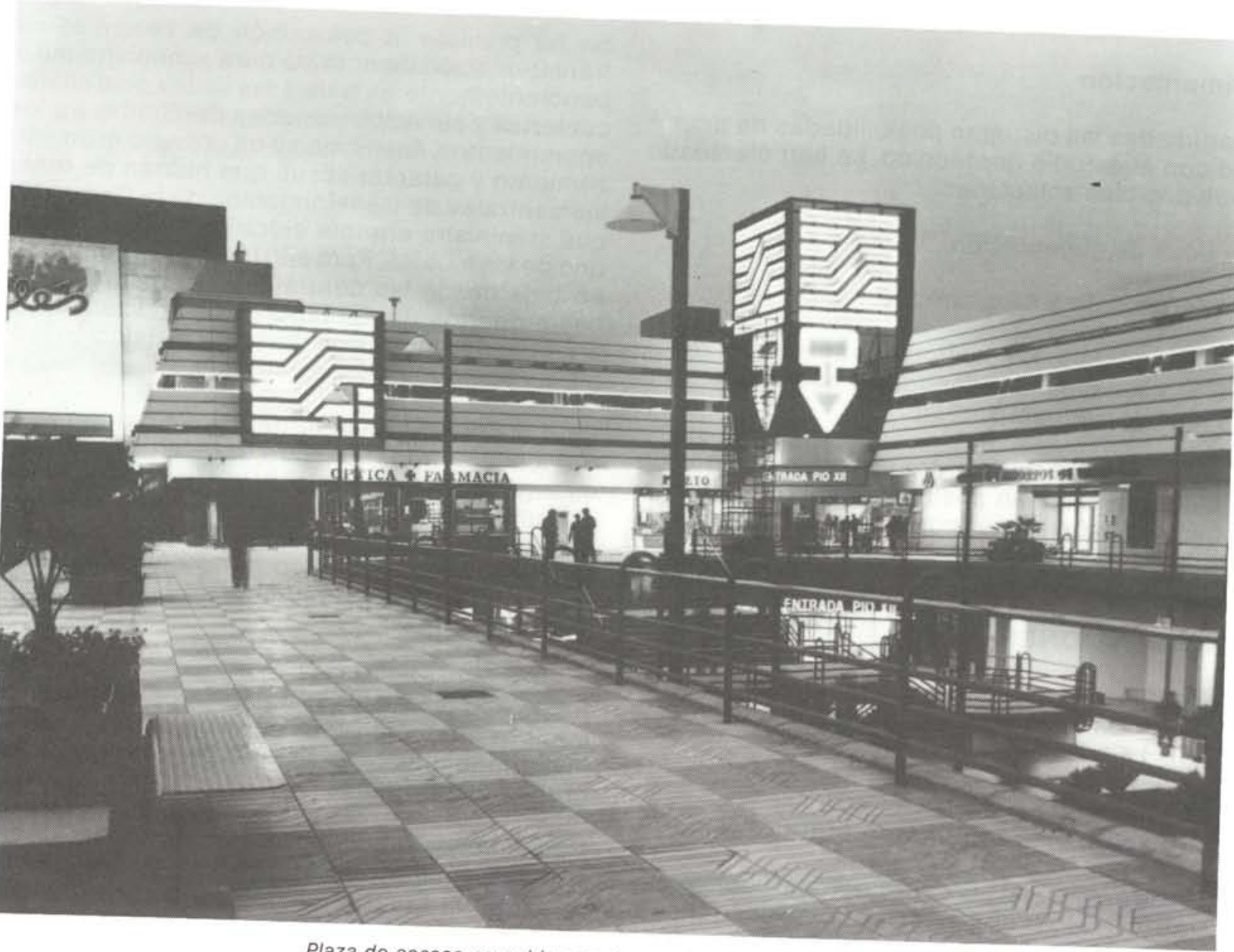

Plaza de acceso con el hueco de penetración a la planta -4 


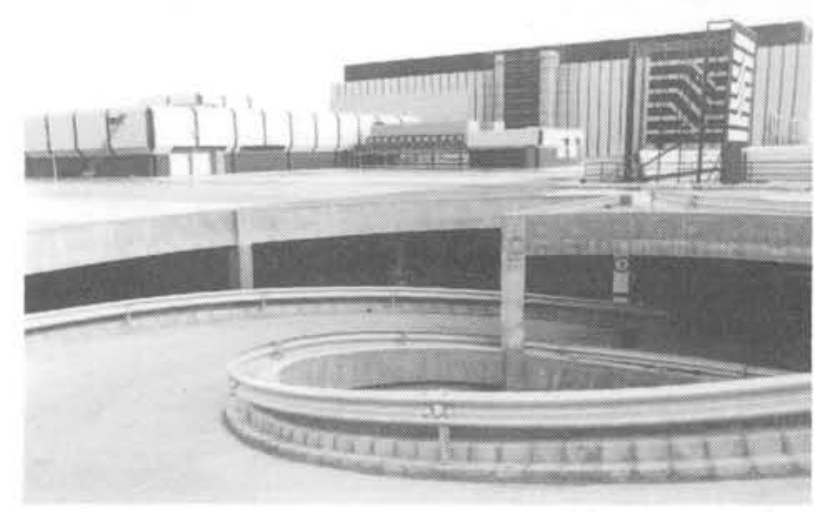

Rampa helicoidal de subida a los aparcamientos.

Los soportes, por razones de dilatación y rapidez de ejecución, son de hormigón en la planta inferior y en la formación de rampas para vehículos, siendo metálicos en el resto del edificio, excepto en zona de aparcamiento.

\section{Carga y sobrecargas}

Se han considerado en la estructura las cargas y sobrecargas que fija la norma MV-101.

\section{Cimentación}

Estudiadas las distintas posibilidades de acuerdo con el estudio geotécnico, se han planteado tres posibles soluciones:

- Losa de cimentación.

- Pilotaje.

- Zapatas de $2,5 \mathrm{~kg} / \mathrm{cm}^{2}$.

Se ha adoptado la solución de zapatas por resultar funcionalmente adecuada y económicamente más conveniente.

Las zapatas se unen entre sí mediante vigas de atado que eliminan posibles desplazamientos y absorben momentos producidos en especial en las zapatas de borde.

\section{INSTALACIONES}

A continuación se realiza una sucinta descripción de las instalaciones comprendidas en esta FASE 1.a.

\section{Ventilación y acondicionamiento}

Dada la amplitud de la calle peatonal cubierta, se ha estudiado la ventilación y acondicionamiento de ésta, mediante una instalación de aire acondicionado de volumen variable en función de la (c) Consejo Superior de Investigaciones Científicas ocupación de cada área que suministra aire exterior suficiente, tanto para la propia calle peatonal como para los locales que se abren en ella a efectos de crear el ambiente de calle con temperatura y humedad controladas. La ventilación del conjunto calle-locales se complementa por medio de una red de conductos de extracción que, tomando el aire de los locales y áreas comunes lo echa al exterior mediante extractores centrifugos.

La carga interna de los locales se toma con unidades autónomas colocadas por el propio usuario y condensada por agua, para lo cual se dispone de un circuito cerrado general conectado a una torre de refrigeración en cubierta.

La ventilación del aparcamiento subterráneo se realiza mediante ventiladores de impulsión y extracción que barren con aire exterior toda el área del mismo.

Los ventiladores antes citados están activados automáticamente por un sistema de detección de CO, capaz de dar alarma si los niveles de monóxido alcanzan cotas peligrosas en algún punto del aparcamiento.

\section{Electricidad, fuerza y alumbrado}

Se ha previsto la colocación de centrales de transformación de energia para suministro independientemente en baja a las calles peatonales cubiertas y servicios comunes del Centro y a los aparcmientos. Asimismo se ha previsto el emplazamiento y características que habrán de tener las centrales de transformación de la Compañía que suministra energía eléctrica en baja a cada uno de los locales. Para ello hay líneas generales en baja desde las centrales de transformación hasta cuartos de contadores centralizados por áreas, desde las que se realiza la instalación propia de cada uno de los usuarios.

Para el servicio de emergencia se han previsto grupos electrógenos que dan alumbrado de emergencia a la calle peatonal cubierta, áreas comunes de servicio y áreas exteriores, así como potencia para los servicios preferentes del Centro. Todo ello se pone en funcionamiento automáticamente al faltar suministro eléctrico de la Compañía.

Se ha completado la instalación con una red de equipos autónomos con acumulador que cubren instantáneamente el tiempo de entrada de los grupos electrógenos.

Para emergencia de los locales, aparte de los anteriores equipos, se ha previsto en los grupos electrógenos una potencia equivalente a $3 \mathrm{~W} / \mathrm{m}^{2}$ de local para suministrarles acometida de emergencia a los mismos. http://informesdelaconstruccion.revistas.csic.es 


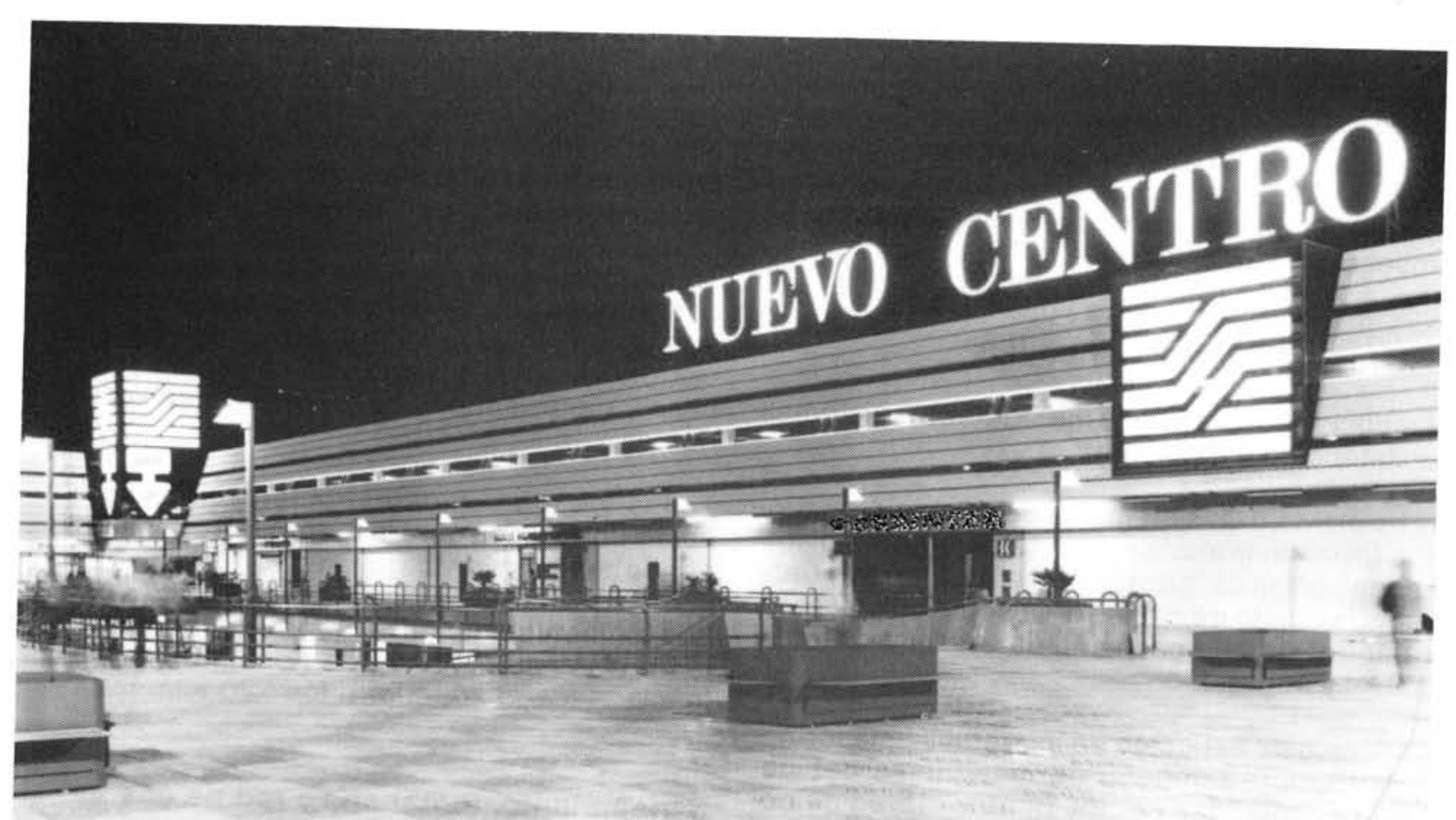

Vista de la plaza de acceso.

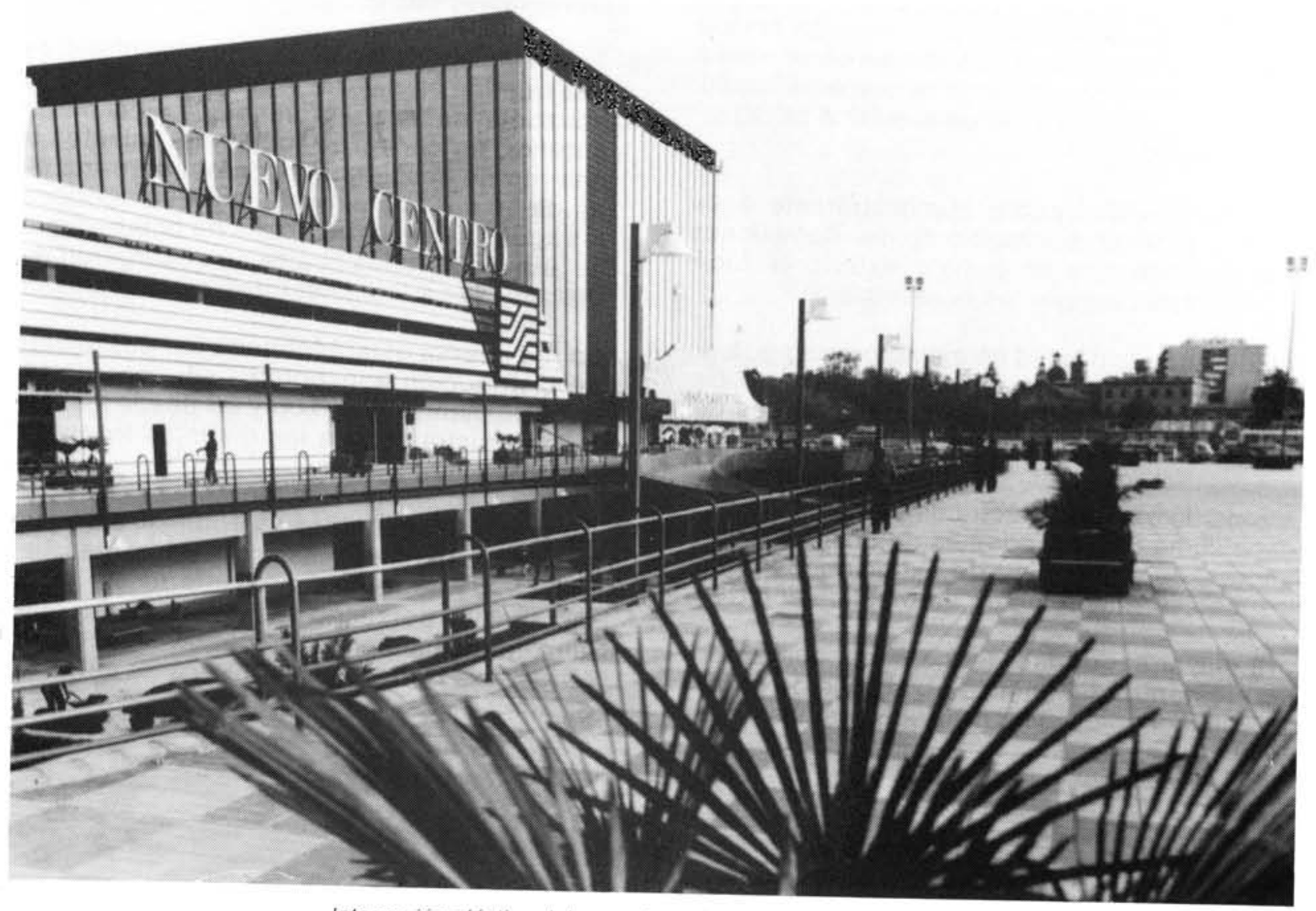

Integración plástica del gran almacén en el área comercial. 
Las intensidades de iluminación de calle peatonal cubierta y plazas, se conseguirán con equipos fluorescentes, de halógenos e incandescentes en cada zona para conseguir una iluminación media adecuada a normas según el uso, reforzando los puntos focales con complementos de proyección y luz indirecta.

\section{- Instalaciones contra incendios}

El edificio está dotado de los siguientes sistemas de detección y extinción:

Se ha previsto un sistema de detección de incendios formado por una malla de detectores distribuidos por las calles peatonales cubiertas y tiendas, con objeto de dar la señal acústico-visual en la central de alarmas, situada en un cuarto de control y seguridad.

El sistema de extinción de incendios está formado por una red general de mangueras debidamente distribuidas por los puntos claves de comunicación vertical, calles peatonales interiores, áreas de servicios comunes y aparcamientos, alimentadas por un anillo de agua (red interior de incendios) que se encuentra siempre a la presión de servicio gracias a los grupos de bombeo que para tal fin se han previsto.

El caudal de agua necesario se suministra mediante dos fuentes principales (depósito reserva de $828 \mathrm{~m}^{3}$ de capacidad y alimentación directa de la red urbana) que se consideran como inagotables y otra fuente secundaria que está constituida por un depósito hidroneumático de $30 \mathrm{~m}^{3}$ de capacidad.

Al anillo principal podrá suministrársele agua desde el exterior, por medio de dos conexiones siamesas situadas en puntos exteriores fácilmente accesibles por los bomberos.

Colaborando con la red de extinción por agua, se han previsto extintores portátiles y de carro, debidamente distribuidos por todas las áreas del Centro y aparcamientos, según normas.

La zona de cuadros eléctricos y grupos electrógenos se ha dotado, además de una red de detectores, de un sistema de extinción de incendios mediante inundación automática con $\mathrm{CO}_{2}$.

Para mayor seguridad del personal que se encuentre dentro del edificio en el caso de producirse un incendio, se ha previsto, además de los sistemas de detección y extinción, un procedimiento de desalojo mediante salidas de emergencia debidamente distribuidas y señalizadas que, comunicadas directamente con el exterior, permitan un rápido desalojo del Centro y los aparcamientos.

Todos los pasillos disponen de puertas metálicas cortafuegos con elemento de cierre automático y señalización de función sobre la misma. Asimismo, disponen de bisagras de vaivén en zona de paso y cierre antipánico de presión en las exteriores.

Todos los elementos estructurales de soporte están protegidos contra fuego de 120 minutos y tienen revestimiento decorativo exterior.

Los elementos de cerramiento y separación están previstos en bloque de hormigón con resistencia al fuego superior a los 120 minutos.

\section{Saneamiento, fontanería y red de gas}

El sistema de desagües para el edificio se ha diseñado en dos escalones bien diferenciados:

A) Se recogen las aguas pluviales procedentes de niveles de rasante y cubiertas por techo de planta cota 95, mediante una red colgada que canaliza por gravedad las aguas limpias al colector municipal.

B) Todas las aguas fecales y aquellas pluviales que queden en niveles inferiores al de la red horizontal de recogida, señalada en el punto anterior, se canalizan mediante bajantes a una red de desagüe horizontal enterrada situada en cota de cimentación que conduce las aguas a los cuatro pozos de bombeo que se han previsto, los cuales se encargarán de desaguarlas a los colectores municipales.

La red interior estará formada por dos anillos de distribución horizontales situados en techos de plantas cota 100 y 95 desde los que se suministra agua a los distintos locales y servicios.

Se suministra gas al área del edificio en la que existe demanda del mismo (restaurantes, bares, etc.), y se realiza mediante un anillo de distribución por techo de planta cota 95, que, alimentado mediante acometida de la red exterior de la Compañía, cubre el área antes indicada. 